\title{
Morphological Modification of Poly (ethylene terephthalate) Surface after Excimer Laser Irradiation
}

\author{
Wilson Wong ${ }^{1}$, Kwong Chan ${ }^{1}$, Kwok Wing Yeung ${ }^{1}$ and Kai Shui Lau ${ }^{2}$ \\ 1. Institute of Textile \& Clothing, The Hong Kong Polytechnic University, Hong Kong. \\ 2. Department of Applied Physics, The Hong Kong Polytechnic University, Hong Kong.
}

\begin{abstract}
Poly (ethylene terephthalate) was modified by a $\mathrm{KrF}$ excimer laser $248 \mathrm{~nm}$. It was observed that the PET surface developed a very often periodic roughness or ripple. The roughness size is in the micron range and the surface shows some sign of global melting Irradiated samples were imaged and studied using atomic force microscopy (AFM) and scanning electronic microscopy (SEM). AFM was used to follow the morphological changes caused by subsequent laser pulses on the very same area of the irradiated PET samples. AFM also provided quantitative data on the laser induced surface and allowed three-dimensional imaging of nonconducting surfaces. Changes in surface morphology of PET fibres were found in relation to laser energy applied. The mean roll to roll distance increased with increasing laser energy. Merging of ripples was observed and believed to be a major reason of increased roll to roll distance. With approximately 50 to 200 pulses, ripple almost approached parallelism. No further change of PET surface was observed with more laser pulses applied since the fibre has disintegrated into "ellipsoidal segments". Meanderlike structures superimposed onto the top of the ripples were observed. The wetting property of PET due to laser treatment was also studied.

(Received Nov. 22, 1999)

(Accepted for Publication March 9, 2000)
\end{abstract}

\section{Introduction}

In the last decade, many efforts have been made in developing surface treatments such as UV irradiation and lamp, plasmas, flame, corona, electron beams and ion beams [1]. UV laser modification on materials surface is one of the most studied technologies. It has been known that materials like polymers, woods, metals, semiconductors, dielectrics, quartz modified by UV laser are often accompanied by physical as well as chemical changes of the materials surface. In case of polymer, some well oriented structures of hills and grooves or ripple structures in the range of micron are developed on the surface with irradiation fluence above the so-called ablation threshold e.g. PET irradiated by $248 \mathrm{~nm}=30 \mathrm{~mJ} / \mathrm{cm}^{2}[2-3]$. The threshold values depend on the wavelength of the UV irradiation and the absorption coefficient of the polymer. These induced structures show some interesting characteristics. For example, they are strictly perpendicular to the stress direction of the materials and strongly influenced by laser wavelength and energies, absorption coefficient etc [4-7]. The formation of the ripple is believed to be due to the synergetic phenomena $[2-3,8]$. Because of the extremely high absorption coefficient of the polymer to the laser energy deposition, releasing of the frozen-in tension fields; resulting in a self-organizing material convection. However, only a small area can be 
irradiated/treated at one time because of the relatively small cross-section of excimer laser beam and the relative high value of the ablation threshold required. These induced structures could increase the value of polymer such as better adhesion and dyeability [9-10]. In addition laser treatment is essentially a dry process that

\section{Experimental}

\subsection{Laser treatment}

Poly (ethylene terephthalate) samples both fibre and fabric were irradiated in air with a commercial Lambda Physik COMP EX 205 makes it possible to alter the surface properties of the materials while maintaining desirable bulk properties intrinsic to polymers. Laser treatment does not involve any solvents unlike the wet chemical process, and thus eliminates environmental pollution.

excimer laser. The experiment setup is shown in Figure 1. The laser operates with $\mathrm{KrF}$ gas and produces wavelength $\lambda=248 \mathrm{~nm}$. The pulse repetition rate was kept constant at $1 \mathrm{~Hz}$ to avoid heat accumulation. Laser energies like fluence and number of pulse vary from experiments in order to study their effects upon samples.

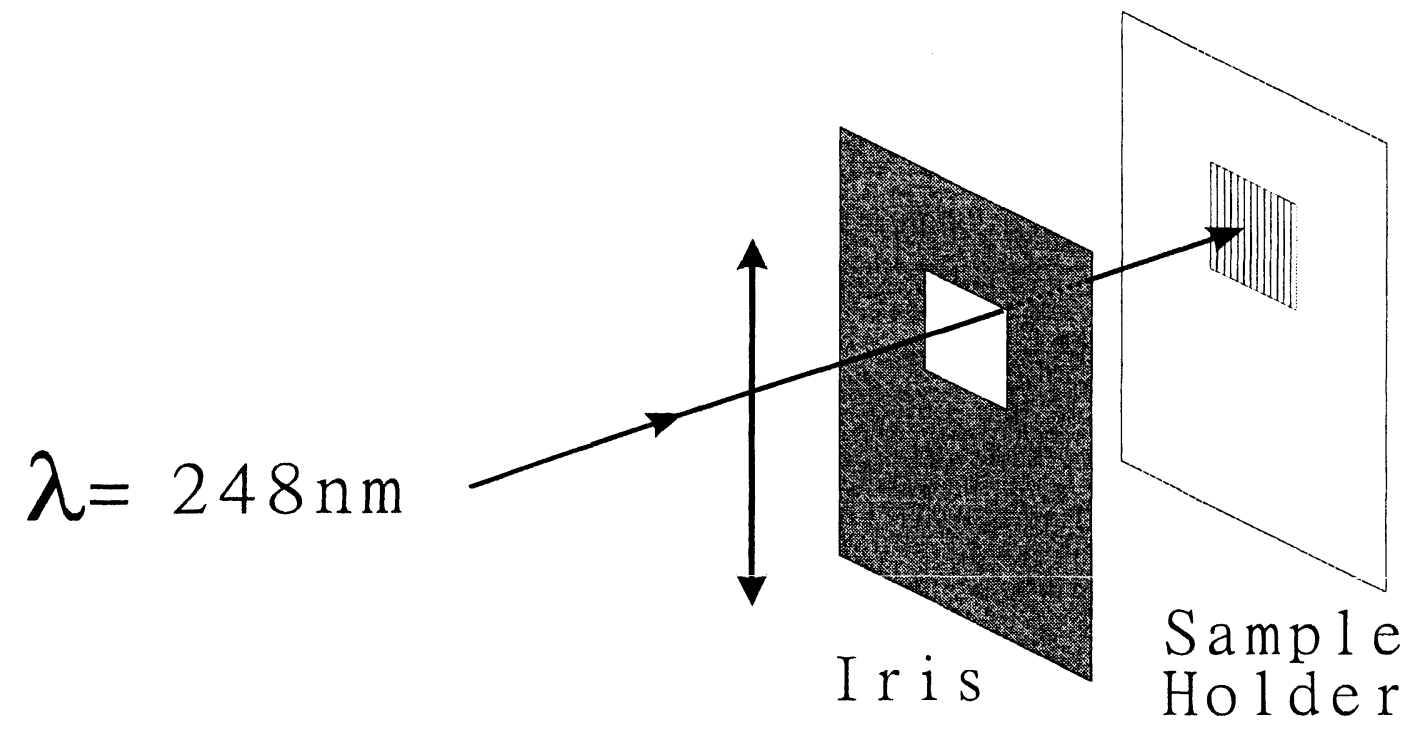

Figure 1 Experimental setup for laser irradiation

\subsection{Morphological study}

The AFM imaging was conducted under atmospheric pressure with a commercial scanning probe microscope (Metris-2000 buleigh). Imaging was acquired in contact mode and at constant force. This was achieved during scanning in $\mathrm{x}$ and $\mathrm{y}$ directions by rearranging the piezoelectric in $\mathrm{z}$ direction. The maximum piezodrive scan in the $\mathrm{z}$ direction was limited to
$6 \mu \mathrm{m}$. Hence, it was not possible to profiling the whole fibre and only the most elevated part was imaged. In order to maximize the positioning reproducibility of the head to the irradiated area, a high-resolution optical microscope together with a special marking were used. Samples viewed by SEM (Lecia Stereoscam 440) were gold coated prior to examination. 


\subsection{Vertical drop test}

Vertical drop test was conducted in accordance with BS 4554 (1970). A drop of distilled water is allowed to fall onto a fabric and the time, $T_{s}$, for which the liquid is required to sink into the fabric completely, i.e. the liquid is no longer visible from the surface of the fabric, was recorded. The shorter the time, the more wettable is the fabric. Fabric with time exceeding 200s is considered to be unwettable for hydrophilic materials whereas wetting time will be longer if hydrophobic material is used.

\section{Results and Discussion}

\subsection{Morphological Study}

Figure 2 presents a set of AFM images made on the PET fibre. As can be seen, the surface of the untreated PET sample shows only minor irregularities (within $60 \mathrm{~nm}$ ) but no recognizable damages and this irregularities is believed to be connected with manufacturing defects. With only one laser pulse of $50 \mathrm{~mJ} / \mathrm{cm}^{2}$, the surface topography changed dramatically from an original smooth surface to a fibrous surface characterized by hills and grooves. The orientation of this newly developed structure is rather weak. The second laser pulse of the same fluence has transformed these hills and grooves to a more uniform one, which is usually known as ripple-like structure. With four laser pulses, the surface becomes coarser and the z-average has increased from the $260 \mathrm{~nm}$ in the second pulse to $650 \mathrm{~nm}$. In addition, the mean roll distance also increased. After 16 pulses (Figure 2e), a transition of the small ripple into a welldeveloped one was noticed. The topography has not been changed much with further laser pulses since it is believed the evolution and development of this structure depends mainly on the structure after the first pulse. However, a change in surface roughness was detected. The surface roughness computed from AFM images are summarized in Figures 3 and 4 . The $R_{a}$ (variance of the surface) is calculated using Equation 1 shown below [11].

$$
\frac{1}{N} \sum_{I}^{N}\left|Z_{i}-Z_{\text {avg }}\right|
$$

As can be seen from Figures 3 and 4 that both $R_{a}$ and ripple spacing show a positive relation with laser energy i.e. $R_{a}$ and ripple spacing increase with the number of pulses. For the $R_{a}$, the value increased dramatically after the second pulse and slow down afterwards. An increase in $R_{a}$ represents an increase in ripple size. The reason of the increased ripple spacing is largely due to the merging of the ripples (Fig. 5a) [12]. Since the enlargement of ripple is come from the material self-movement. More laser energy would give a larger distance between hills. With approximately 50 to 200 pulses (depends on materials and laser energy), ripple almost approached saturation (see $5 \mathrm{~b}$ ). It is hard to notice further change of PET surface with more laser pulses since the fibre has disintegrated into "ellipsoidal segments" (see 5c). Meander-like structure superimposed onto the top of the ripples was observed (see 5d). 


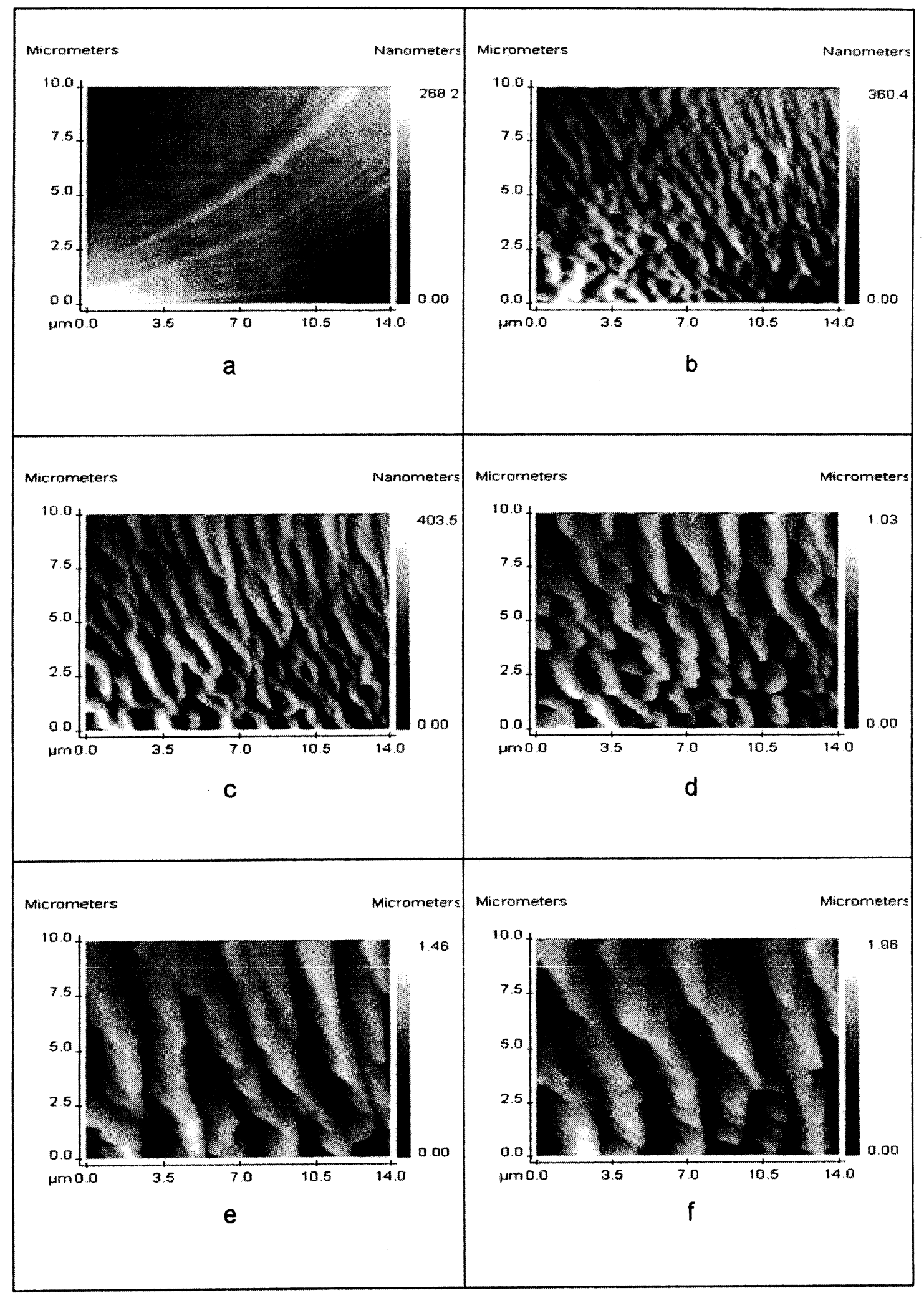

Figure 2 A series of AFM images of PET fibre treated with laser irradiation (Fluence $=50 \mathrm{~mJ} / \mathrm{cm}^{2}$ ) at different laser pulses: $\mathrm{a}=$ untreated, $\mathrm{b}=1$ pulse, $\mathrm{c}=2$ pulses, $\mathrm{d}=4$ pulses, $\mathrm{e}=16$ pulses and $\mathrm{f}=32$ pulses. 


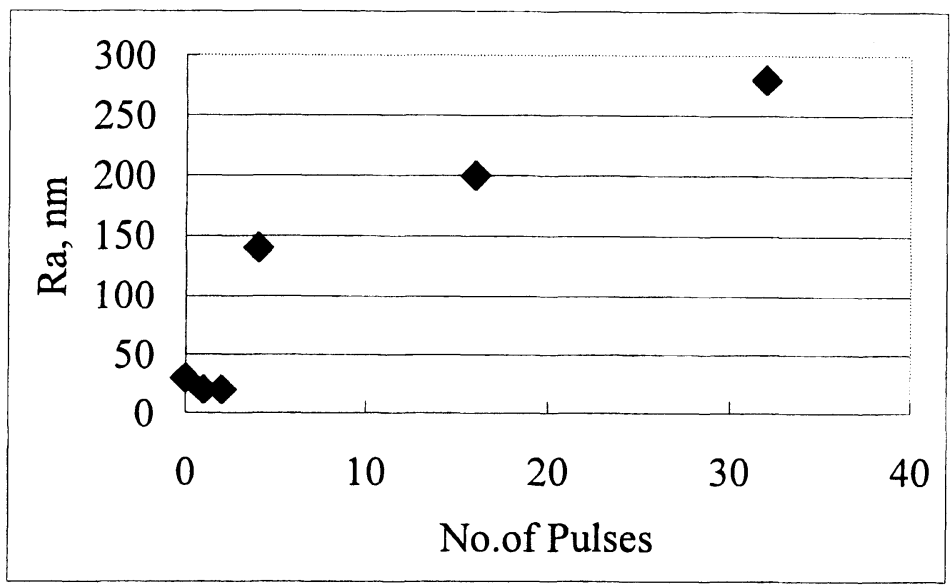

Figure 3 Roughness study of laser treated PET

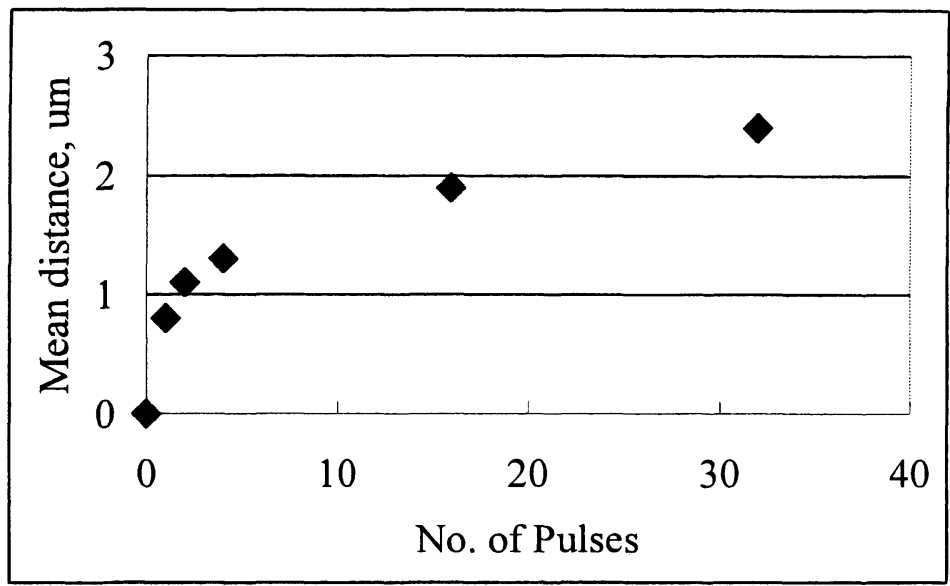

Figure 4 Ripple study of Laser treated PET
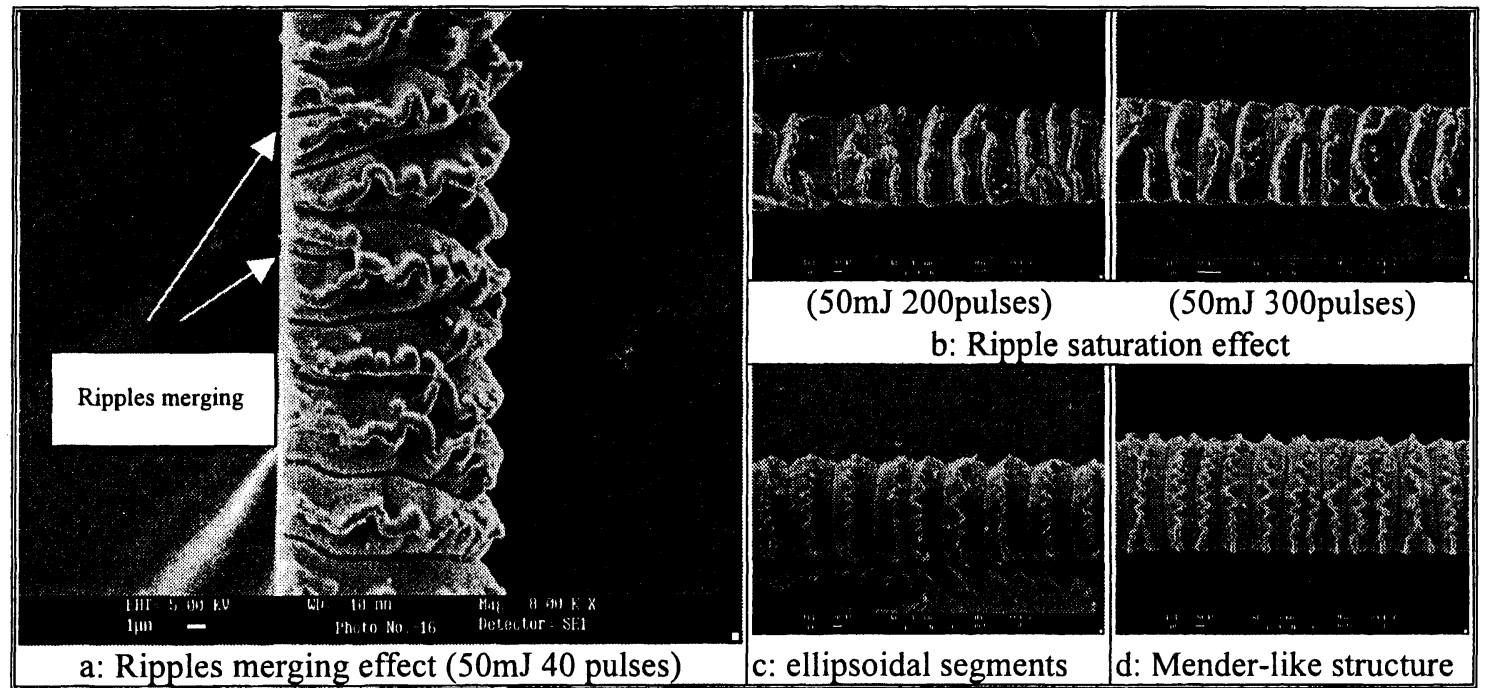

a: Ripples merging effect ( $50 \mathrm{~mJ} 40$ pulses)

c: ellipsoidal segments

d: Mender-like structure

Figure 5 SEM images: a- Ripples merging resulting in an increase of the ripple spacing, b- No further change of fibre surface once ripple saturated, c- ellipsoidal segments, and dMeander-like structure superimposed onto the top of the ripples.

3.2 Vertical drop study

PET fabric sample was used in this study. $\mathrm{T}_{\mathrm{s}}$, the time for which the liquid is required to sink into the fabric completely, of the fabric before and after laser treatment $\left(70 \mathrm{mj} / \mathrm{cm}^{2} .10\right.$ pulses $)$ 
are shown in Table 1. The results of the vertical drop test show that laser treatment increases the wetting time of the laser irradiated sample greatly. The reason is believed to be due to the fact that ripple structure on the surface of the irradiated fabric enhances the surface roughness of the fabric. This kind of increased roughness enhances the unwettability of the fabrics, resulting in an increase in the wetting time. Researchers have experimentally proved that if the surface is sufficiently rough, a liquid with a large contact angle may not completely wet the surface. This incompletely wetted surface provides rooms for air to be trapped between the liquid and solid interface, resulting in a so-called composite interface [12] in preventing water penetration. In addition, surface of aromatic polymer like PET will normally result in the deposition of some yellow to black materials, often known as debris, after laser treatment. These debris are ionized and carbon rich materials which finally condenses forming higher aggregates [13-14], resulting in a more hydrophobic surface [15]. Figure 6 show the difference in $T_{s}$ of the measurement for laser treated fabric sample, on which the left half of the fabric was treated by laser.

Table 1 Results of the vertical drop test before and after laser treatment

\begin{tabular}{lcc}
\hline & Before Laser Treatment & After Laser Treatment \\
\hline Fabric & $470 \mathrm{~s}$ & $>900 \mathrm{~s}$ \\
\hline
\end{tabular}

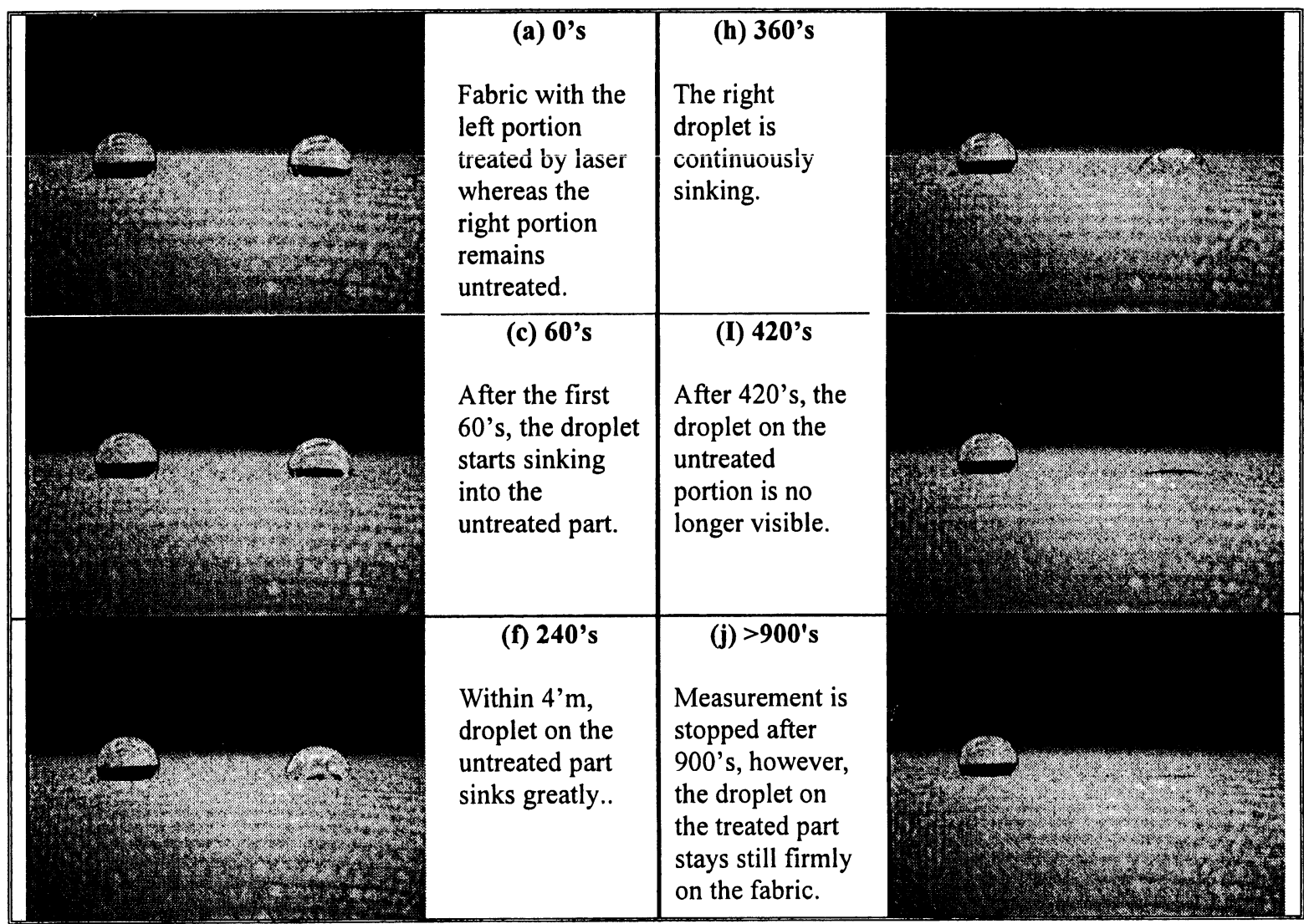

Figure 6 The effect of Laser treatment $\left(70 \mathrm{mj} / \mathrm{cm}^{2} .10\right.$ pulses $)$ on wetting time 


\section{Conclusion}

It has been shown that the use of laser upon PET is a valuable tool to modify surface properties of the materials. Surface roughness and ripple spacing increased with laser energy. Merging of the ripples is the driven force for the increased ripple spacing. With the appropriate laser treatment, the hydrophobicity of polyester could be greatly enhanced, making the treated polyester highly unwettable and this provides the textile industry a new method in making hightech water repellent textile product.

\section{Acknowledgement:}

The Authors would like to thank Dr. G.K.H. Pang for AFM images. The work described in this paper was supported by The Hong Kong Polytechnic University Research Grant (A/C V518)

\section{Reference:}

[1] Chan C.M., Ko T.M. and Hiraoka H., Surface Science Report 24 (1996) 1-54.

[2] Knittel D., Kesting W. and Schollmeyer E., Polymer International, 43 (1997) 231-239.

[3] Knittel D., Kesting W. and Schollmeyer E., Polymer International, 43 (1997) 240-250.
[4] Schollmeyer E., AATCC Book of Papers, Eng. ed., Oct. (1995) 353-358.

[5] Srinivasan R. and Braren B., Chemical Reviews, (1989) 89 1303-1316.

[6] Barnnon J.H., Lankard J.R., Baise A.I., Burns F. and Kaufman J., Journal Applied Physics, 58 (5) (1985) 2036-2043.

[7] Lazare S., Soulignac J.C. and Fragnaud P., Applied Physics Letters, 50 (10) (1987) 624-625.

[8] Bahners $\mathrm{T}$ and Schollmeyer E., Journal Applied Physics, 66 (4) (1989) 1884-1886.

[9] Lau K.S., Chan P.W., Wong K.H., Yeung K.W., Chan K., and Gong W.Z., Journal Materials Processing Technology, 63 (1997) 524.

[10] Murahara M. and Okoshi M., Polymer Surface Modification: Relevance to Adhesion, Part 1 (1996) 223.

[11] Dowdy S.M., Statistics for research, New York: Wiley, (1991).

[12] Chan C. M., Polymer Surface Modification and Characterization, Hanser Publishers, (1994) 34.

[13] Knittel D. and Schollmeyer E., Angew. Makromol. Chem., 178 (1990) 143.

[14] Koren G. and Yeh J.T.C., Journal Applied Physics, 56 (1984) 2120.

[15] Knittel D. and Schollmeyer E., Polymer International, 45 (1) (1998) 103. 